\title{
Vergleich der Wirkung der explorativen Tympanoskopie inkl. Obliteration der runden/ovalen Fensternische mit autologem Bindegewebe mit intratympanalen Kortikoidinjektionen bei der Behandlung des Hörsturzes
}

\subsection{Einleitung}

\subsubsection{Hinführung zum Thema}

Die intra-/transtympanalen Steroidinjektionen (ITST) stellen unseres Erachtens das Verfahren in der Behandlung des Hörsturzes dar, das in seinem Indikationsbereich mit dem der explorativen Tympanoskopie einschließlich Abdichtung des runden/ovalen Fensters (TYMP) am ehesten verglichen werden kann und zur Zeit sicher von beiden Methoden weltweit (noch) am häufigsten im Second-Line-Vorgehen Verwendung findet. Deshalb sollen in diesem Kapitel die aus der Literatur ableitbaren Hördaten beider Therapieregimes einander gegenübergestellt werden.

\subsubsection{Formen der ITST- und Tympanoskopietherapie}

Die ITST können als alleinige Therapiemaßnahme oder aber, wie zumeist auch die explorative Tympanoskopie, zusätzlich zu einer parenteralen/oralen und damit systemischen Verabreichung der Kortikosteroide durchgeführt werden. Je nachdem, ob die ITST/TYMP zeitlich parallel zu der systemischen Kortikoidbehandlung abläuft oder der intravenösen/oralen Gabe folgt, bezeichnet man die Therapieform als simultan (englisch ,concommitant“, „concurrent“ oder „,combined“) oder sie kommt sequenziell (englisch „,subsequent“) zur Anwendung. 


\subsubsection{Metaanalysen und systematische Reviews bei der ITST- und Tympanoskopietherapie}

In der Literatur findet man eine Vielzahl von Publikationen, die sich in Form statistisch anspruchsvoller Metaanalysen dem Erfolg der ITST widmen [20, 27, 41, 51, 68, 76, 80, 110, 112]. Im Gegenteil dazu konnten wir keinen, mit vergleichbaren statistischen Methoden aufgearbeiteten Beitrag entdecken, der sich auf die Tympanoskopie mit Abdeckung des runden/ovalen Fensters bei der Behandlung des Hörsturzes bezieht. Diesem Mangel soll in diesem Beitrag, der in Form eines systematischen Reviews gestaltet wird, zumindest in einer statistisch weniger anspruchsvollen Weise begegnet werden, wobei auch die Daten eines eigenen Patientengutes mit in die Auswertung einfließen.

\subsection{Patientengut und Methode}

\subsubsection{Filterkriterien zur Studienauswertung}

Als Filterkriterien, um in die vorliegende Auswertung aufgenommen zu werden, galten über diejenigen, die den Hörsturz an sich definieren, hinaus [1]:

- Vorhandensein audiologischer Daten vor Therapiebeginn (in der Regel zum Zeitpunkt der stationären Krankenhausaufnahme oder direkt prätherapeutisch) und am Ende einer Nachbeobachtungszeit von mindestens 3 Wochen nach ITST/TYMP.

- Alternative Bewertung der Hörgewinne nach den modifizierten KanzakiKriterien [35].

- Anfangshörverlust von >50 dB bei den Tympanoskopierten.

- Bei den intratympanalen Kortikoidinfusionen Berücksichtigung von mittleren Hörverlusten, die mit einer PTA 4 -Methode $(0,5,1,2$ und $4 \mathrm{kHz}$ oder 0,5, 1, 2 und $3 \mathrm{kHz})$ bzw. PTA 5 -Methode $(0,25,0,5,1,2$ und $4 \mathrm{kHz})$ bestimmt wurden. Wir hielten es für angemessen, auch eine Studie mit einer Bewertung der Hörerfolge nach modifizierten Kanzaki-Kriterien basierend auf einer $\mathrm{PTA}_{3}$ Bestimmung $(0,5,1$ und $2 \mathrm{kHz})$ in die Auswertung aufzunehmen [16].

Bezüglich des Zeitintervalls, das zwischen dem Abschluss der stationären Krankenhausbehandlung und dem Nachuntersuchungstermin vorausgesetzt wurde, kann auf eine publizierte Studie und eigene Untersuchungen verwiesen werden, die gezeigt hatten, dass sich einen Monat nach Beendigung der Therapie im weiteren Zeitverlauf bis zu einem später erfolgten Nachuntersuchungstermin (2,3 Jahre) keine weiteren wesentlichen Hörveränderungen (lediglich $>5 \mathrm{~dB}$ durchschnittlicher Hörgewinn) mehr einstellen ([60], eigene Studie). Bei dem Vorgehen mit intratympanalen Kortikoidapplikationen (ITST) lagen dazu vergleichbare Beobachtungen vor $[16,45,100,104]$. 


\subsubsection{Rekrutierung des Patientengutes}

Insgesamt beinhalten 23 Publikationen 34 Studiengruppen, bei denen eine ITSTisoliert, kombiniert oder sequenziell durchgeführt worden war, und es gibt 4 Veröffentlichungen mit 5 Studiengruppen zum Thema TYMP (Tab. 8.1) [29, 30, 33 , 97]. Hinzugefügt werden konnten 3 Studiengruppen aus unserem eigenen Krankengut, die bisher noch nicht veröffentlicht sind (Gesamtzahl an Patienten: $\mathrm{n}=2170)$ (Tab. 9.1).

\subsubsection{Arten der Therapiedurchführung und Rekrutierung des Patientengutes}

Es ließen sich neben der systemischen Applikation von Steroiden (SST) drei Verfahrensweisen herausarbeiten, nach denen die ITST bzw. auch die Tympanoskopie im Rahmen des Hörsturzes zur Anwendung kamen (Tab. 9.1 und Tab. 9.2)

- Alleinige systemische orale oder i.v.-Applikation von Steroiden (SST-Gruppe).

- Alleinige intratympanale Steroidinjektionen (ITST-Gruppe).

Tab. 9.1 Rekrutierung des Patientengutes aus der Literatur zum Vergleich der Wirkung der intratympanalen Steroidinjektionen und der Tympanoskopie. SST = alleinige systemische Kortikoidbehandlung; ITST = alleinige intratympanale Steroidinjektionen; $C O M B=$ synchrone systemische Kortikoidbehandlung und intratympanale Steroidinjektionen; $S E Q=$ sequenzielle systemische Kortikoidbehandlung mit nachfolgenden intratympanalen Steroidinjektionen; $T Y M P=$ Tympanoskopie, entweder in synchroner Behandlung mit systemischen Steroiden oder als sequenzielle Behandlung mit anfänglich systemischen Steroiden und nachfolgender Operation

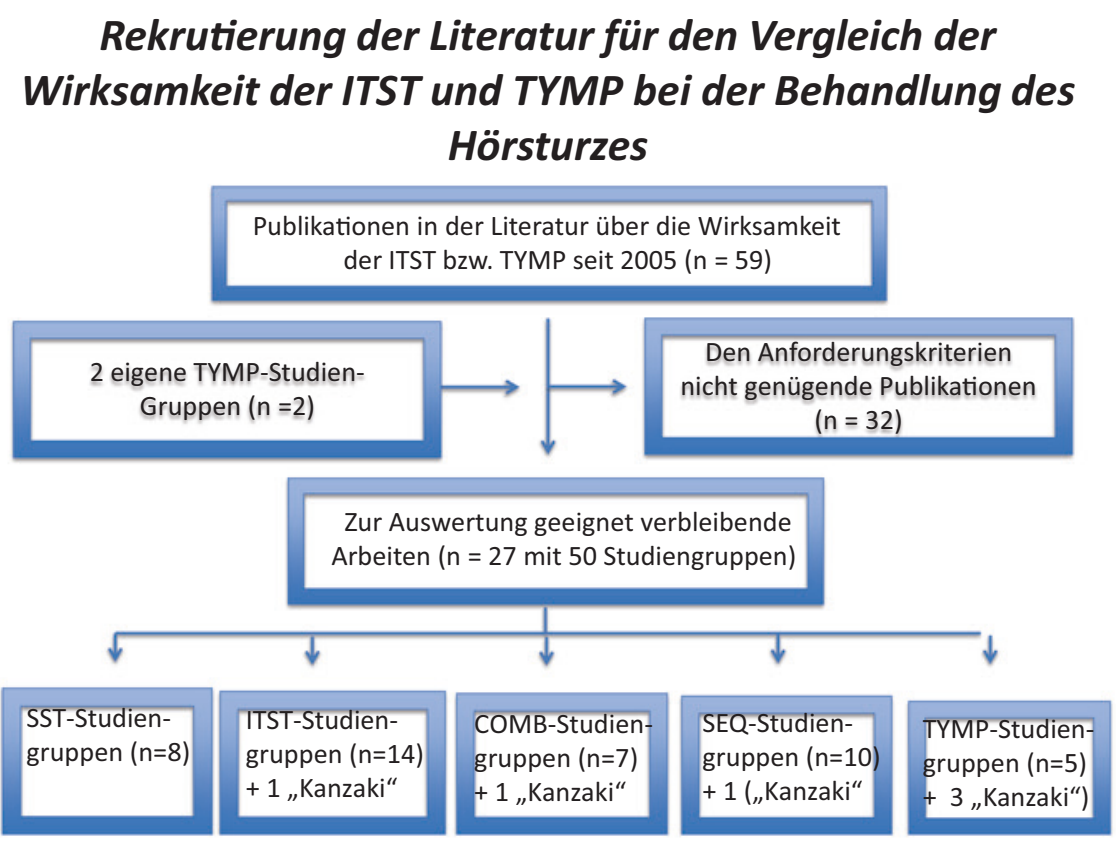




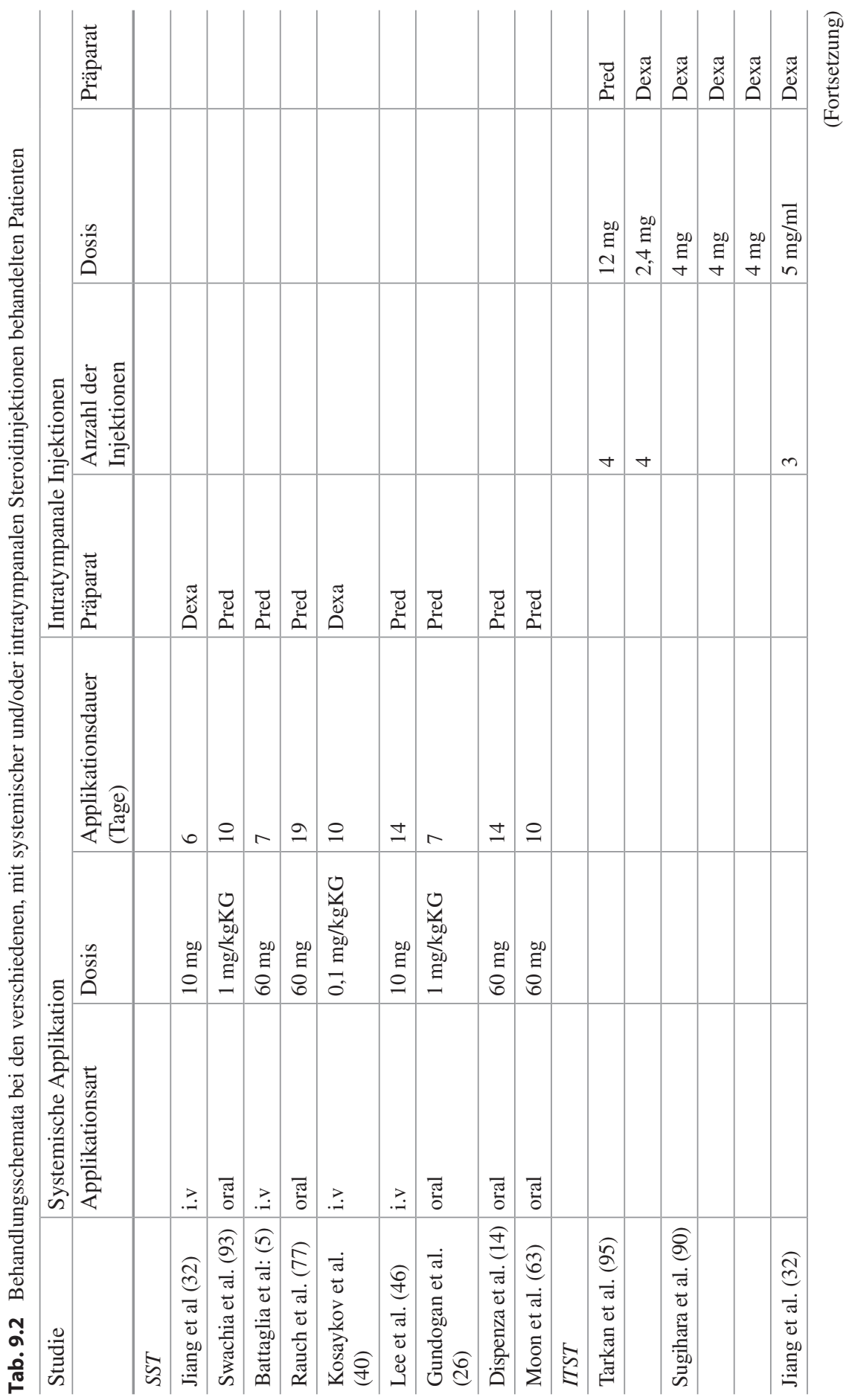




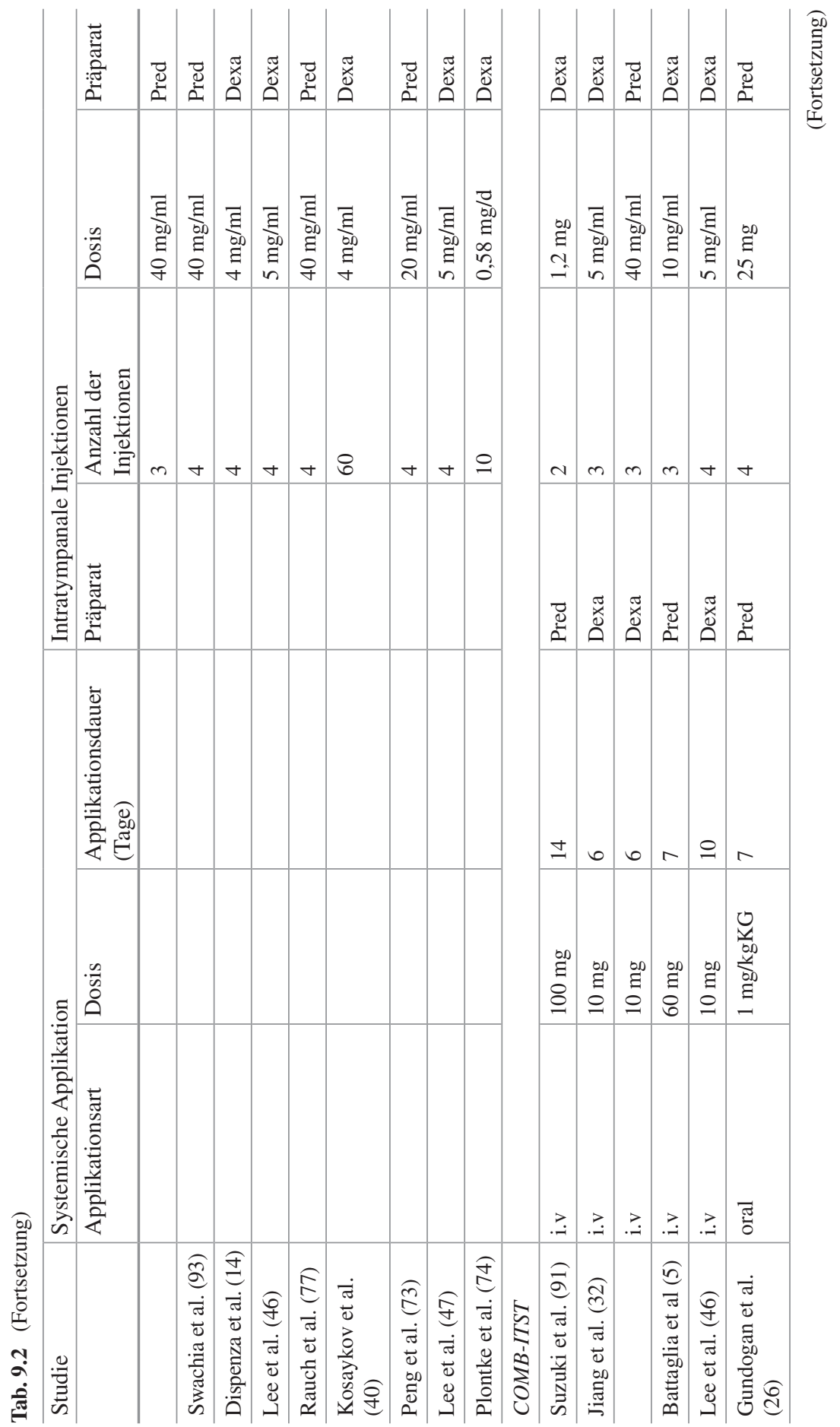




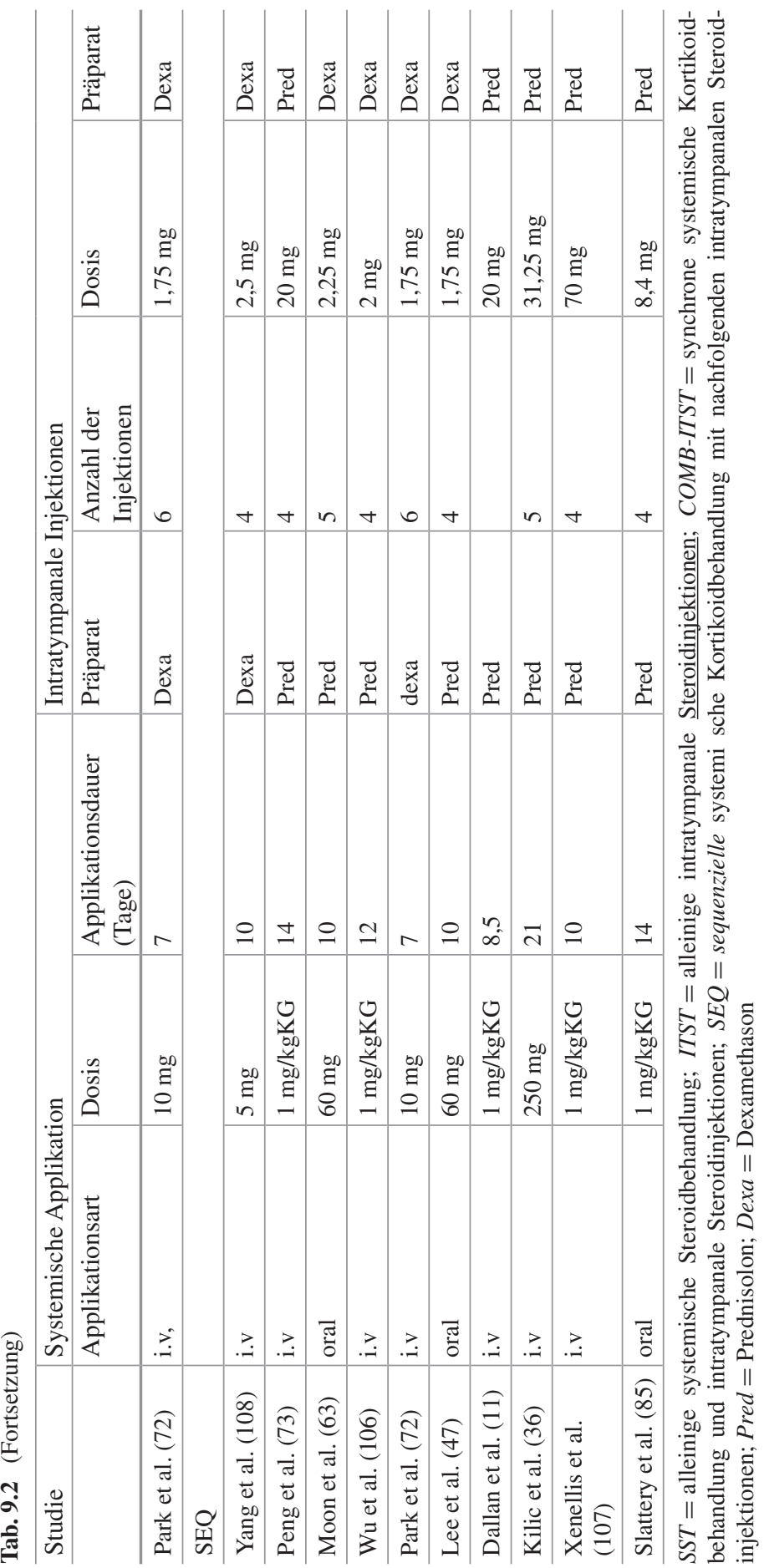


- Zeitlich früh (First-Line-Modus) eingesetzte intratympanale Steroidinjektionen bzw. durchgeführte Tympanoskopien synchron mit einer systemischen Kortikoidapplikation (COMB-ITST-Gruppe und COMB-TYMP-FL-Gruppe).

- Zeitlich verzögert/sequenziell durchgeführte intratympanale Steroidinjektionen bzw. Tympanoskopien nach einer vorherigen systemischen Kortikoidtherapie (SEQ-Gruppe resp. TYMP-SL-Gruppe).

Eine gewisse Anzahl von Arbeiten, die sich auch diesem Thema gewidmet haben, konnte nicht in die Auswertung aufgenommen werden $(n=32)$, da entweder die Frequenzzusammensetzung, aufgrund derer ein mittlerer Hörverlust ermittelt worden war, nicht den von uns gesetzten Kriterien entsprach, die Nachbeobachtungszeit der Patienten weniger als 3 Wochen betrug (in den Publikationen lediglich als "posttherapeutisches/postoperatives Ergebnis“ bezeichnet), die Latenzzeit zwischen Hörsturzereignis und Tympanoskopie zu lang war (>10 Tage) oder aber das Gesamtpatientenkollektiv in Untergruppen aufgeteilt worden war, die dann jeweils gesondert bezüglich ihres Hörgewinns untersucht worden waren, ohne dass eine Mitteilung über die Ergebnisse des Gesamtkollektivs erfolgte.

\subsubsection{Art der Kortikoidapplikation}

Die systemische Kortikoidapplikation erfolgte in 6 Studiengruppen oral und bei 27 Patientenkollektiven parenteral. Einigen Patientengruppen waren zusätzlich noch Vasodilatantien, Vitamine und/oder Pentoxifyllin gegeben worden.

Gewöhnlich wurden bei der ITST 4 Steroidinjektionen mit bis zu $5 \mathrm{mg}$ Dexamethason oder $1 \mathrm{mg}$ Prednisolon/kg Körpergewicht über einen Zeitraum von etwa 2 Wochen intratympanal injiziert/infundiert (bei Katheterlage), wobei die Perforation des Trommelfells im hinteren unteren Quadranten unter Lokalanästhesie erfolgte (Tab. 9.2).

\subsubsection{Technik der Tympanoskopie}

Die Tympanoskopien folgten den Regeln einer routinemäßig durchgeführten Mittelohroperation. Haubner et al., Hoch et al. sowie Kampfner et al. dichteten, unabhängig von der Tatsache, ob eine Perilymphabfluss gesehen werden konnte, nur die runde Fenstermembran mit autologem Bindegewebe ab, Thomas et al., wie auch wir, obliterierten beide Fensternischen [29, 30, 33, 97]. 


\subsubsection{Prospektive und randomisierte Studien}

Es wurden pro- und retrospektive, randomisierte und nichtrandomisierte sowie (Doppel-)Blindstudien als auch nichtverblendete Patientenkollektive in die Auswertung aufgenommen.

\subsubsection{SST-Gruppen}

Die Patienten der SST-Gruppe rekrutierten sich aus den einzelnen Studien, die über die intratympanalen Steroidinjektionen berichtet hatten und die dort sozusagen als Kontrollgruppe dienten. Aus 8 Studien konnten auf diese Weise 385 Patienten (345 Patienten mit Dezibelangaben der Hörverluste) rekrutiert werden (Tab. 9.1, Tab. 9.2 und Tab. 9.4).

\subsubsection{ITST-Gruppen}

Alleinige intratympanale Kortikoidinjektionen (ITST-Gruppe) hatten 574 Patienten erhalten (525 Patienten mit Dezibelangaben der Hörverluste; zusätzlich 49 Patienten mit Hörgewinnangaben nach den modifizierten Kanzaki-Kriterien), die aus 10 Publikationen (15 Studien) zusammengeführt werden konnten (Tab. 9.1, Tab. 9.2, Tab. 9.3 und Tab. 9.5). Der Nachuntersuchungstermin lag im Mittel 3,8 Monate nach der intratympanalen Steroidbehandlung.

\subsubsection{COMB-ITST-Gruppen}

Eine synchrone Behandlung in Form von systemischen Kortikoidgaben und gleichzeitiger Verabreichung intratympanaler Glukokortikoidinjektionen (COMBITST-Gruppe) war bei 437 Patienten (437 mit Dezibelangaben der Hörverluste; 104 Patienten mit Hörgewinnangaben nach modifizierten Kanzaki-Kriterien), die sich auf 7 Studien (6 Publikationen) verteilten, erfolgt (Tab. 9.1, Tab. 9.2, Tab. 9.3, Tab. 9.6). Die Kontrolluntersuchung fand durchschnittlich 1,9 Monate nach der Hörsturzbehandlung statt.

\subsubsection{SEQ-Gruppen}

Bei 338 Erkrankten (306 Patienten mit Dezibelangaben der Hörverluste; zusätzlich 32 Patienten mit Hörgewinnangaben nach Kanzaki-Kriterien) folgten die intratympanalen Kortikoidinjektionen sequenziell der systemischen Kortikoidbehandlung (SEQ-Gruppe). Diese Patienten wurden aus 11 Studien (11 Publikationen) zusammengetragen (Tab. 9.1, Tab. 9.2, Tab.9.3 und 9.7). Die abschließende Höruntersuchung folgte im Mittel 1,6 Monate nach der intratympanalen Steroidbehandlung. 
Tab. 9.3 Beschreibung der Studiengruppen, die in die Auswertung der Hörgewinne nach der modifizierten Kanzaki-Methode Eingang fanden

\begin{tabular}{|c|c|c|c|c|c|}
\hline Studiengruppe & Publikationsjahr & Ort & $\begin{array}{l}\text { Patienten- } \\
\text { zahl }\end{array}$ & $\begin{array}{l}\text { Kompletter } \\
\text { Hörgewinn } \\
(\mathrm{dB})\end{array}$ & $\begin{array}{l}\text { Markanter } \\
\text { Hörgewinn } \\
\text { (dB) }\end{array}$ \\
\hline \multicolumn{6}{|l|}{ ITST } \\
\hline Kosaykov et al. (40) & 2011 & Moscow & 24 & 60 & 20 \\
\hline \multicolumn{6}{|l|}{ COMB-ITST } \\
\hline Suzuki et al. (92) & 2016 & Kitakiyushu & 197 & 19,1 & 45,2 \\
\hline \multicolumn{6}{|l|}{$S E Q$} \\
\hline Ermutlu et al. (16) & 2017 & Istanbul & 32 & 37,5 & 11,4 \\
\hline \multicolumn{6}{|l|}{ TYMP } \\
\hline Eichhorn & 2020 & Cottbus & 42 & 24,7 & 35,6 \\
\hline Thomas et al. (97) & 2018 & Bochum & 136 & 28 & 36,8 \\
\hline Hoch et al. (30) & 2015 & Marburg & 51 & 23,5 & 21,4 \\
\hline
\end{tabular}

$I T S T=$ Intratympanale Kortikoidinjektionen; $\quad C O M B-I T S T=$ Synchrone systemische Kortikoidtherapie und intratympanale Kortikoidinjektionen; $S E Q=$ sequenzielle Applikation einer systemischen Kortikoidgabe und nachfolgend intratympanale Kortikoidinjektionen; TYMP$S L=$ sequenzielle Therapie mit anfänglich systemischen Kortikoidgaben und nachfolgender explorativer Tympanoskopie

\subsubsection{TYMP-Gruppen}

Aus 6 Studien (4 Publikationen) und der Hinzufügung der von uns behandelten Patienten in 3 Studiengruppen ließ sich ein Krankengut von Hörgeminderten zusammenstellen, das entweder dem Therapiemodus eines synchron kombinierten (COMB-TYMP-FL-Gruppe; $\mathrm{n}=79$ ) oder aufeinander folgenden, sequenziellen (TYMP-SL-Gruppe =298; 247 Patienten mit Dezibelangaben der Hörverluste; zusätzlich 51 Patienten mit Hörgewinnangaben nach modifizierten Kanzaki-Kriterien) Vorgehens der explorativen Tympanoskopie bezogen auf die systemische Kortikoidtherapie entsprach (Tab. 9.1, Tab. 9.3 und Tab. 9.8).

Insgesamt flossen in die nachfolgende Auswertung die Hördaten von 2117 Patienten ein.

\subsection{Ergebnisse}

\subsubsection{Variationsbreiten der Anfangs- und Endhörverluste}

\subsubsection{Vorgehensweise der Auswertung}

Aufgrund des vorliegenden Datenmaterials schien es uns angemessen, zuerst nur Variationsbeschreibungen bezüglich der in den einzelnen Studien angegeben Hörbefunde darzustellen und erst in einem zweiten Auswertungsschritt gewichtete 


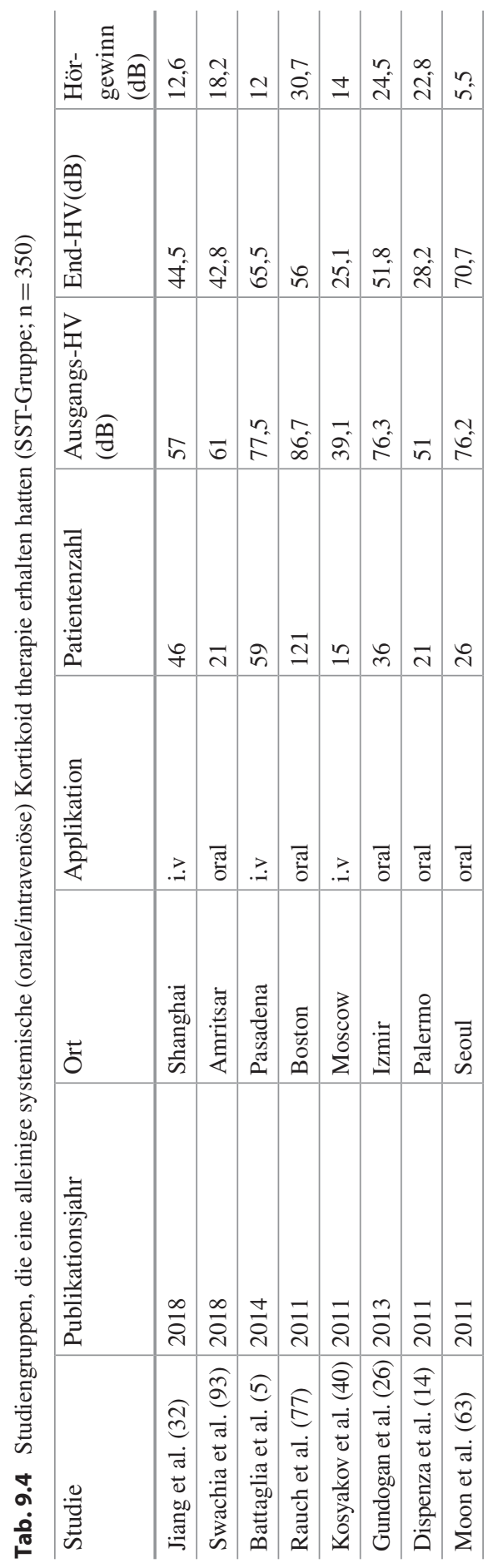


Mittelwertbildungen vorzunehmen, ohne weitergehende Signifikanzberechnungen in die Auswertung einfließen zu lassen.

\subsubsection{SST-Gruppe}

Bei der systemischen Applikation von Kortikosteroiden lagen die Ausgangshörwerte zwischen 39,1 dB und 86,7 dB (Tab. 9.4 und Abb. 9.1). Am Ende der Therapie betrugen die Hörverluste noch zwischen 25,1 dB und 70,7 dB. Es hatten sich Hörgewinne zwischen 5,5 dB und 30,7 dB eingestellt.

\subsubsection{ITST-Gruppe}

Der kleinste, in einer Studiengruppe gemessene Hörverlust, der bei diesen Patienten, die eine alleinige intratympanale Steroidinjektionen erhalten hatten, ermittelt wurde, betrug 41,0 dB, der höchste 98,5 dB (Tab. 9.5 und Abb. 9.1). Bei der Kontrolluntersuchung wurden Werte zwischen 16,1 dB und 75,6 dB gemessen. Die Hörgewinne lagen zwischen 10,2 dB und 41,8 dB.

\subsubsection{COMB-ITST-Gruppe}

In der COMB-ITST-Gruppe, bei der die systemische Kortikoidapplikation zeitlich parallel zu den intratympanalen Injektionen erfolgt war und wo beide Maßnahmen zeitnah am Hörsturzereignis lagen, betrugen die Hörverlust bei der stationären Krankenhausaufnahme zwischen 62,8 dB und 84,8 dB (Tab. 9.6 und Abb. 9.1). Bei den Follow-up-Untersuchungen schwankten die zuletzt gemessenen Werte zwischen $36,6 \mathrm{~dB}$ und $55,1 \mathrm{~dB}$. Die Hörverbesserungen bewegten sich in der Spanne zwischen $16,8 \mathrm{~dB}$ und $44,1 \mathrm{~dB}$.

\subsubsection{SEQ-Gruppe}

Bei den Patienten der SEQ-Gruppe, bei denen die ITST der systemischen Steroidapplikation sequenziell folgte, wurden Ausgangswerte zwischen 60,7 dB und 94,6 dB gemessen (Tab. 9.7 und Abb. 9.1). Im Rahmen der letzten Kontrolle wurden Resthörverluste zwischen 39,7 dB und 75,7 dB eruiert. Die Hörgewinne betrugen zwischen 7,3 dB und 32,6 dB.

\subsubsection{COMB-TYMP-FL-Gruppe}

In die Bestimmung der Variationsbreiten der COMB-TYMP-FL-Gruppe gingen nur die Ergebnisse von zwei Studiengruppen ein. Die Ausgangshörverluste lagen zwischen 93,1 dB und 106,1 dB (Tab. 9.8 und Abb 9.9). Die Höreinschränkungen bei der Nachuntersuchung betrugen $67,8 \mathrm{~dB}$ bzw. 71,1 dB. Als Hörgewinne konnten minimal $22 \mathrm{~dB}$ resp. maximal $32,8 \mathrm{~dB}$ verzeichnet werden.

\subsubsection{TYMP-SL-Gruppe}

Die initialen Hörverlustwerte lagen in der TYMP-SL-Gruppe, bei der die explorative Tympanoskopie der systemischen Kortikoidapplikation folgte, mit Werten zwischen 101,0 dB und 106,5 dB recht dicht beieinander (Tab. 9.8 und Abb. 9.1). Die verbliebenen Höreinschränkungen zum Nachuntersuchungstermin variierten zwischen 42,6 dB und 75,7 dB. Die Spannbreite der Hörerholungen erstreckte sich von $32,8 \mathrm{~dB}$ bis $63,9 \mathrm{~dB}$. 


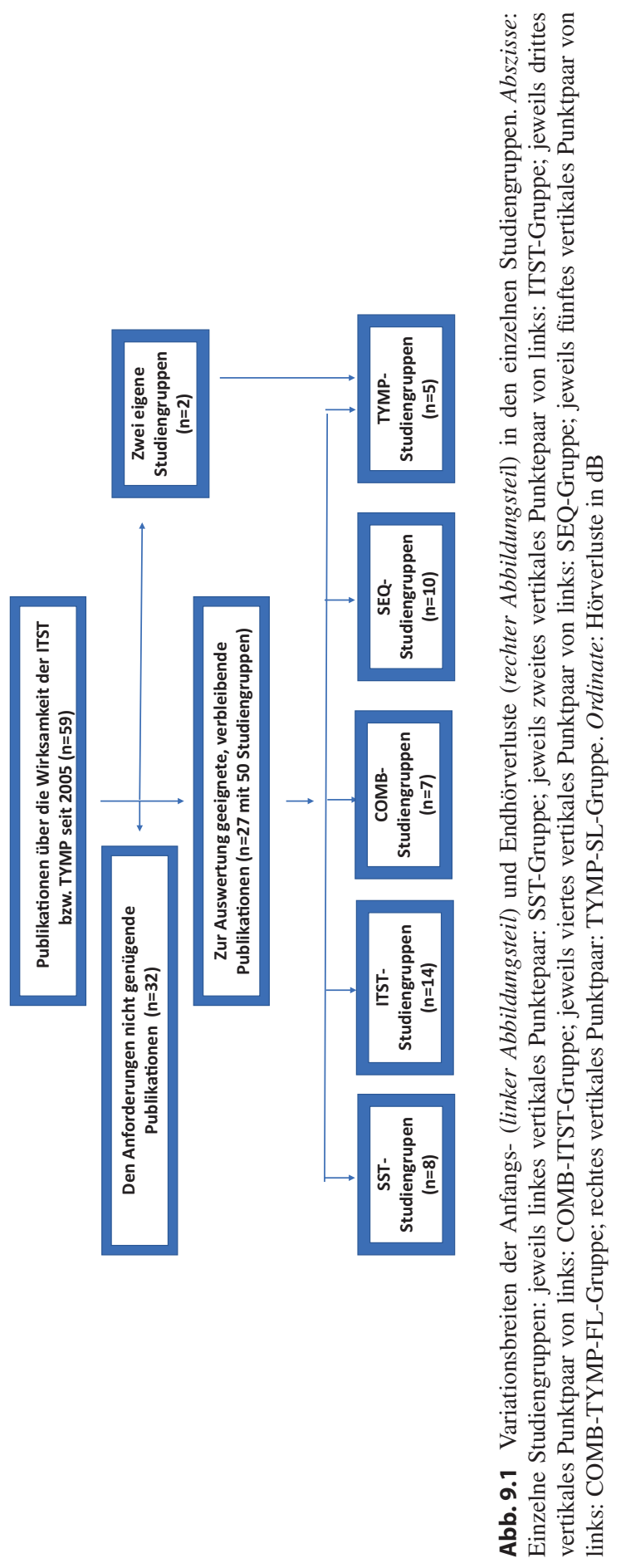


Tab. 9.5 Studiengruppen, die als Therapiemaßnahme intratympanale Steroidinjektionen ohne begleitende systemische Kortikoidgaben erhalten hatten (ITST-Gruppe)

\begin{tabular}{l|l|l|l|l|l|l}
\hline Studie & Publikationsjahr & Ort & $\begin{array}{l}\text { Patienten- } \\
\text { zahl }\end{array}$ & $\begin{array}{l}\text { Anfangs- } \\
\text { HV }(\mathrm{dB})\end{array}$ & $\begin{array}{l}\text { End-HV } \\
(\mathrm{dB})\end{array}$ & $\begin{array}{l}\text { Hör- } \\
\text { gewinn } \\
(\mathrm{dB})\end{array}$ \\
\hline Tarkan et al. (95) & 2018 & Adana & 24 & 71,6 & 40,8 & 30,8 \\
\hline $\begin{array}{l}\text { Sugihara et al. } \\
\text { (90) }\end{array}$ & 2018 & Detroit & 21 & 73,7 & 50,1 & 23,6 \\
\hline & & & 29 & 70 & 54,3 & 19,7 \\
\hline \begin{tabular}{l} 
Jiang et al (32) \\
\hline
\end{tabular} & 2018 & Shanghai & 81 & 65,3 & 55,1 & 10,2 \\
\hline $\begin{array}{l}\text { Swachia et al } \\
\text { (93) }\end{array}$ & 2016 & & 98 & 58,6 & 40 & 18,6 \\
\hline $\begin{array}{l}\text { Dispenza et al. } \\
\text { (14) }\end{array}$ & 2011 & Amritsar & 21 & 66,1 & 51,4 & 14,7 \\
\hline Rauch et al. (77) & 2011 & Palermo & 25 & 65 & 38 & 27 \\
\hline $\begin{array}{l}\text { Kosaykov et al. } \\
\text { (40) }\end{array}$ & 2011 & Boston & 113 & 86,4 & 55,1 & 31,3 \\
\hline \begin{tabular}{l} 
Peng et al. (73) \\
\hline Lee et al. (47)
\end{tabular} & 2011 & Moscow & 24 & 41 & 16,1 & 24,9 \\
\hline $\begin{array}{l}\text { Plontke et al. } \\
\text { (74) }\end{array}$ & 2012 & Guangzhou & 24 & 64,8 & 52,9 & 11,9 \\
\hline
\end{tabular}

Tab.9.6 Studiengruppen, die als Therapiemaßnahme synchron intratympanale Steroidinjektionen mit begleitenden systemische Kortikoidgaben erhalten hatten (COMB-ITST-Gruppe)

\begin{tabular}{l|l|l|l|l|l|l|l}
\hline Studie & Jahr & Ort & $\begin{array}{l}\text { Applikations- } \\
\text { art }\end{array}$ & $\begin{array}{l}\text { Patienten- } \\
\text { zahl }\end{array}$ & $\begin{array}{l}\text { Anfangs- } \\
\text { HV (dB) }\end{array}$ & $\begin{array}{l}\text { End- } \\
\text { HV } \\
\text { (dB) }\end{array}$ & $\begin{array}{l}\text { Hör- } \\
\text { gewinn } \\
(\mathrm{dB})\end{array}$ \\
\hline $\begin{array}{l}\text { Suzuki } \\
\text { et al (92) }\end{array}$ & 2018 & Kitakyushu & i.v & 104 & 77,4 & 49,2 & 28,2 \\
\hline $\begin{array}{l}\text { Jianget al. } \\
\text { (32) }\end{array}$ & 2018 & Shanghai & i.v & 88 & 63,4 & 55,1 & 8,3 \\
\hline $\begin{array}{l}\text { Jiang et al. } \\
\text { (32) }\end{array}$ & 2018 & Shanghai & i.v & 98 & 58,6 & 40 & 18,6 \\
\hline $\begin{array}{l}\text { Battaglia } \\
\text { et al. (5) }\end{array}$ & 2018 & Pasadena & i.v & 80 & 84,8 & 50,6 & 34,2 \\
\hline $\begin{array}{l}\text { Lee et al. } \\
\text { (46) }\end{array}$ & 2015 & Seoul & i.v & 84 & 62,8 & 45,5 & 17,3 \\
\hline $\begin{array}{l}\text { Gundogan } \\
\text { et al. (26) }\end{array}$ & 2013 & Izmir & oral & 37 & 80,7 & 36,5 & 44,1 \\
\hline $\begin{array}{l}\text { Park al. et } \\
\text { (72) }\end{array}$ & 2011 & Seoul & i.v & 44 & 73,1 & 38,4 & 34,7 \\
\hline
\end{tabular}




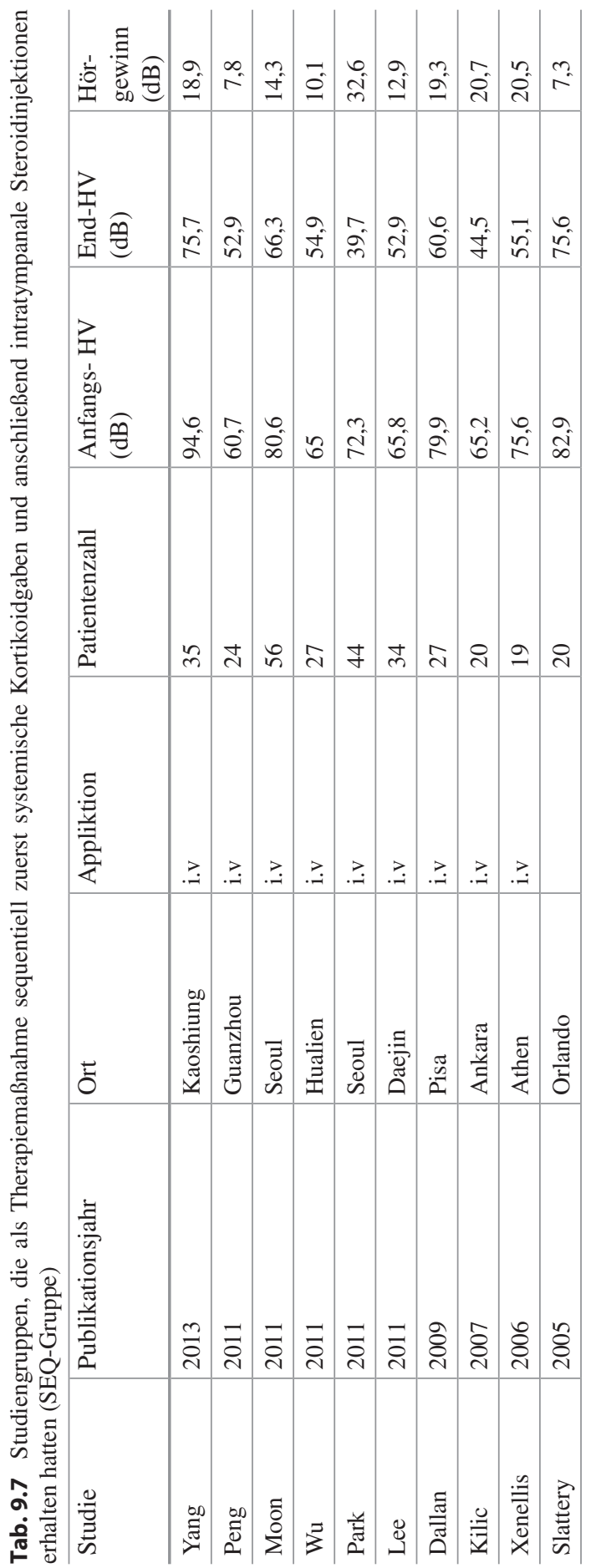




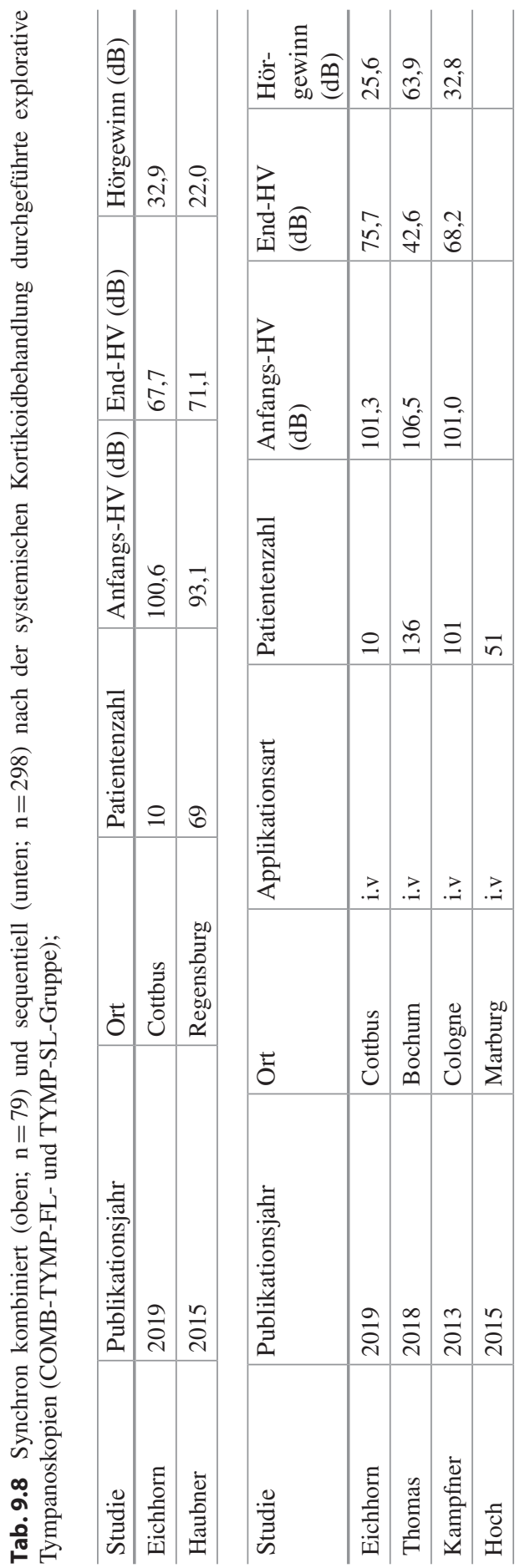




\subsubsection{Vergleich der Variationsbreiten in den Studiengruppen}

Bei den einzelnen Behandlungsverfahren waren bezüglich der tonschwellenaudiometrischen Daten jeweils sehr deutliche Variationsbreiten in den mittleren Anfangs- wie auch Endhörverlusten aufgetreten (Eingangsuntersuchung: $5.5 \mathrm{~dB}$ [TYMP-SL-Gruppe] bis $\quad 57,5 \mathrm{~dB}$ [ITST-Gruppe]; Nachuntersuchung: 8,4 dB [TYMP-SL-Gruppe] bis 59,5 dB [ITST-Gruppe]) (Abb. 9.1). Die mittleren Hörgewinne in Prozent vom Anfangshörverlust lagen zwischen 26,9\% (COMB-ITSTGruppe) und 36,9\% (TYMP-SL-Gruppe).

\subsubsection{Abhängigkeit der Variationsbreiten von der Patientenanzahl}

Wie man auf den beiden Teilen der Abb. 9.1 sehen kann, hängt die Variationsbreite sehr von der Anzahl den in die Auswertung eingebrachten Patientenzahlen ab. Um eine Relativierung der Ergebnisse zu erzielen, wurden deshalb in einem weiteren Analyseschritt die Spannbreiten der Hörverlustwerte durch die Zahlen der eingebrachten Patienten dividiert und damit eine ,relative Variationsbreite“ eruiert. Als Ergebnis sieht man die in Tab. 9.9 eingebrachten Werte. Die geringsten relativen Variationsbreiten bei den Anfangshörverlusten wiesen die TYMP-SLund die COMB-ITST-Gruppen auf (TYMP-SL-Gruppe: 0,02 und COMB-ITSTGruppe: 0,05) und die größten Abweichungen bestanden in der SST-Gruppe $(0,14)$ (Tab. 9.9). Bei den Endhörverlusten zeigten ebenfalls die TYMP-SL- und COMBTYMP-FL-Gruppen die geringsten relativen Spannbreiten (TYMP-SL: 0,03 und COMB-ITST: 0,04) und die SEQ-Gruppe wies die größten relativen Variationsbreiten $(0,19)$ auf.

Aussagekraft der Variationsbreiten Die relativ hohen Variationsbreiten der tonschwellenaudiometrischen Messwerte sowohl bei den initialen wie auch den Endhörverlusten deuten auf eine statistisch labile Datenlage. Abb. 9.1 verdeutlicht aber auch, dass die Patienten mit sehr unterschiedlichen Ausgangshörverlusten in ihre Behandlung ,eingestiegen“ sind, also diesbezüglich nicht von einer Homogenität des untersuchten Krankengutes, insbesondere bei den Studiengruppen mit intratympanalen Kortikoidgaben, ausgegangen werden kann.

Tab.9.9 Variationsbreiten der mittleren Anfangs- und Endhörverluste dividiert durch die Anzahl der in die Berechnung eingegangenen Patientenzahlen (relative Variationsbreiten)

\begin{tabular}{l|l|l}
\hline & Anfangs-HV & End-HV \\
\hline SST & 0,14 & 0,19 \\
\hline ITST & 0,11 & 0,11 \\
\hline COMB-ITST & 0,05 & 0,04 \\
\hline SEQ & 0,12 & 0,13 \\
\hline COMB-TYMP-FL & 0,02 & 0,03 \\
\hline \multicolumn{2}{l|l}{ TYMP-SL } & \multicolumn{2}{l}{} \\
\hline
\end{tabular}

Anfangs- $H V=$ Initialer anfänglicher Hörverlust; End- $H V=$ Endhörverlust zum Nachuntersuchungszeitpunkt 


\subsubsection{Hörentwicklung der gemittelten Anfangs- und Endhörverluste}

\subsubsection{SST-Gruppe}

Bei der Berechnung des gewichteten Mittelwertes aus allen Studiengruppen ergab sich bezüglich dieser Therapieform ein Ausgangshörverlust von 73,5 dB und ein Wert im Rahmen der Nachuntersuchung von 52,9 dB. Im Mittel betrug die Hörverbesserung 20,6 dB (Abb. 9.2).

\subsubsection{ITST-Gruppe}

Als gemittelter Ausgangswert wurde ein Hörverlust von $69,8 \mathrm{~dB}$ berechnet. Die Nachuntersuchung ergab noch eine durchschnittliche Höreinschränkung von 47,3 dB. Damit war ein gemittelter Hörgewinn von 22,5 dB erzielt worden (Abb. 9.3).

\subsubsection{COMB-ITST-Gruppe}

In dieser Studiengruppe lag ein gemittelter Ausgangswert von $73,2 \mathrm{~dB}$ vor. Die Nachuntersuchung ergab noch eine durchschnittliche Höreinschränkung von 41,5 dB. Damit war ein gemittelter Hörgewinn von $31,7 \mathrm{~dB}$ erzielt worden (Abb. 9.4).

\subsubsection{SEQ-Gruppe}

Initial lagen die Höreinschränkungen gemittelt bei $75,2 \mathrm{~dB}$, bei der Nachuntersuchungen bestand noch eine durchschnittliche Höreinschränkung von 56,8 dB.

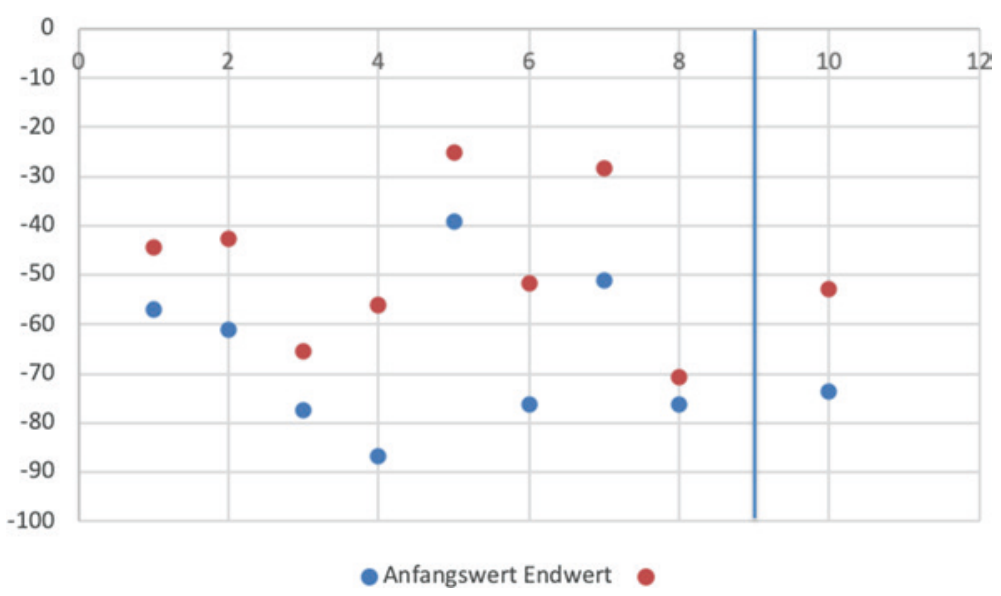

Abb.9.2 Mittlere Tonhörentwicklung der einzelnen SST-Studiengruppen $(\mathrm{n}=345)$. Abszisse: Einzelne Studiengruppen; Ordinate: Hörverluste in dB; rechts neben dem vertikalen Querbalken: gewichteter Mittelwert aus den einzelnen Studiengruppen in dB; unterer Bildpunkt: Anfangshörverlust; oberer Bildpunkt: Resthörverlust bei der Nachuntersuchung 


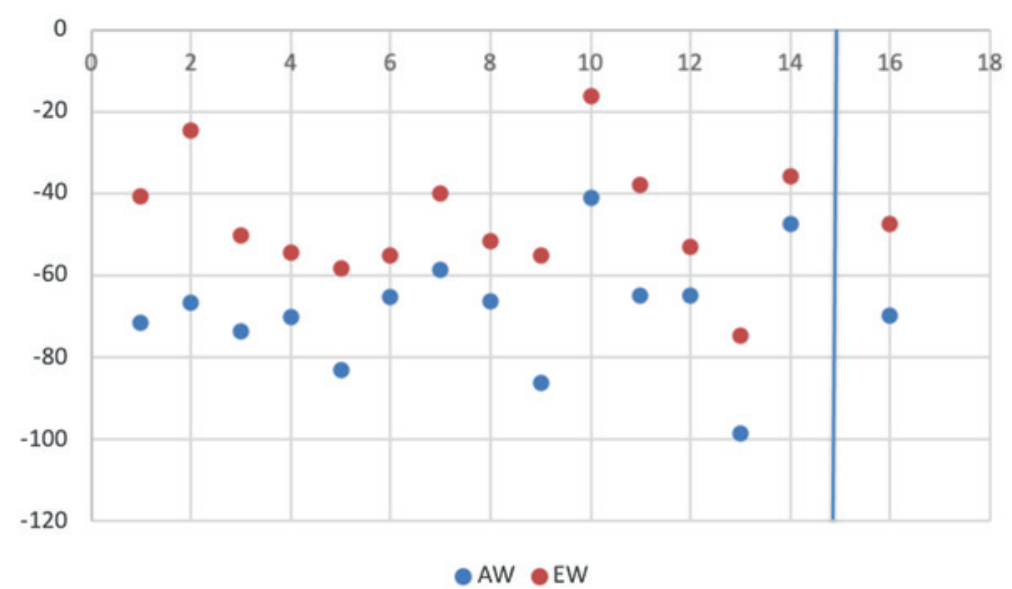

Abb. 9.3 Mittlere Tonhörentwicklung der einzelnen ITST-Studiengruppen $(\mathrm{n}=525)$. Abszisse: Einzelne Studiengruppen; Ordinate: Hörverluste in dB; rechts neben dem vertikalen Querbalken: gewichteter Mittelwert aus den Studiengruppen; unterer Bildpunkt: Anfangshörverlust; oberer Bildpunkt: Resthörverlust bei der Nachuntersuchung.

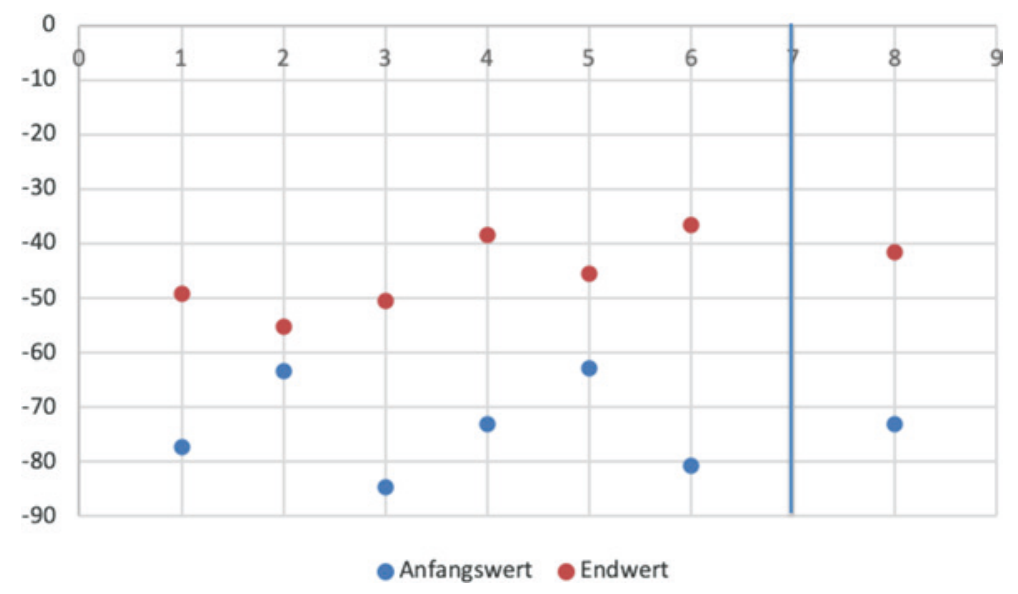

Abb. 9.4 Mittlere Tonhörentwicklung der einzelnen COMB-ITST-Studiengruppen $(\mathrm{n}=$ 437). Abszisse: Einzelne Studiengruppen; Ordinate: Hörverluste in $\mathrm{dB}$; rechts neben dem vertikalen Querbalken: gewichteter Mittelwert aus den Studiengruppen; unterer Bildpunkt: Anfangshörverlust; oberer Bildpunkt: Resthörverlust bei der Nachuntersuchung

Damit war eine Hörverbesserung von 18,4 dB im Mittel zustande gekommen (Abb. 9.5). 


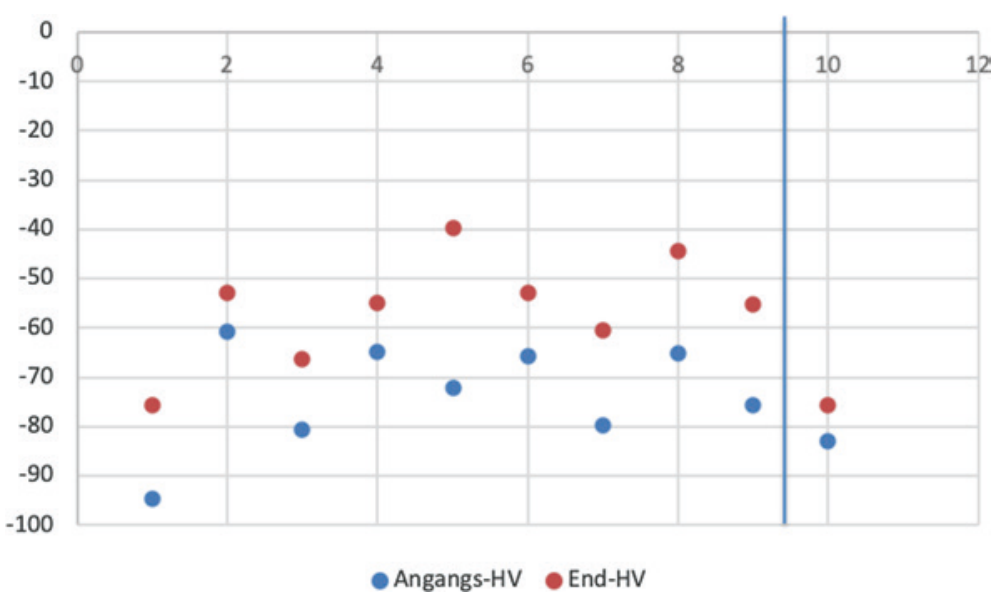

Abb. 9.5 Mittlere Tonhörentwicklung der einzelnen SEQ-Studiengruppen $(\mathrm{n}=306)$. Abszisse: Einzelne Studiengruppen; Ordinate: Hörverluste in $\mathrm{dB}$; rechts neben dem vertikalen Querbalken: gewichteter Mittelwert aus den Studiengruppen; unterer Bildpunkt: Anfangshörverlust; oberer Bildpunkt: Resthörverlust bei der Nachuntersuchung.

\subsubsection{COMB-TYMP-FL-Gruppe}

Die Patienten aus diesen beiden Studiengruppen erschienen mit einer anfänglichen Höreinschränkung von 94,1 dB (Abb. 9.6). Im Rahmen der Nachuntersuchung lag noch eine Höreinbuße von 70,7 dB vor. Es war damit zu einer Hörverbesserung von $23,4 \mathrm{~dB}$ gekommen.

\subsubsection{TYMP-SL-Gruppe}

Die Patienten der TYMP-SL-Gruppe boten anfangs einen Hörverlust von 104,2 dB. Der gemittelte Endhörwert lag bei 66,1 dB. Dem entsprach eine Hörverbesserung von 38,1 dB (Abb. 9.7).

\subsubsection{Vergleich der absoluten und relativen Hörgewinne in den Studiengruppen}

Bezogen auf die absoluten Hörverluste lag die Spanne der Hörverbesserungen zwischen 18,4 dB (SEQ-Gruppe) und 38,1 dB (TYMP-SLGruppe)(Abb. 9.8). Werden die gemittelten Hörgewinne in Prozentangaben auf den Anfangswert bezogen, variierten sie in den verschieden behandelten Studiengruppen zwischen 24,5\% (SEQ-Gruppe) und 43,3 \% (COM-ITST-Gruppe Abb. 9.9 und 9.10).

\subsubsection{Ausgangswerte in den einzelnen Studiengruppen}

Grundsätzlich ist festzuhalten, dass alle Studiengruppen, bei denen Kortikosteroide eingesetzt wurden, deutlich geringere mittlere Ausgangshörverluste aufwiesen, als die Patientenkollektive, die eine Tympanoskopie erhalten hatten (Abb. 9.9). Das gilt sowohl für die Anwendung des operativen Verfahrens im FirstLine- wie auch im Second-Line-Modus. Dies ist insofern nicht verwunderlich, weil die Tympanoskopie aufgrund ihrer traumatisierenden Vorgehensweise bislang 


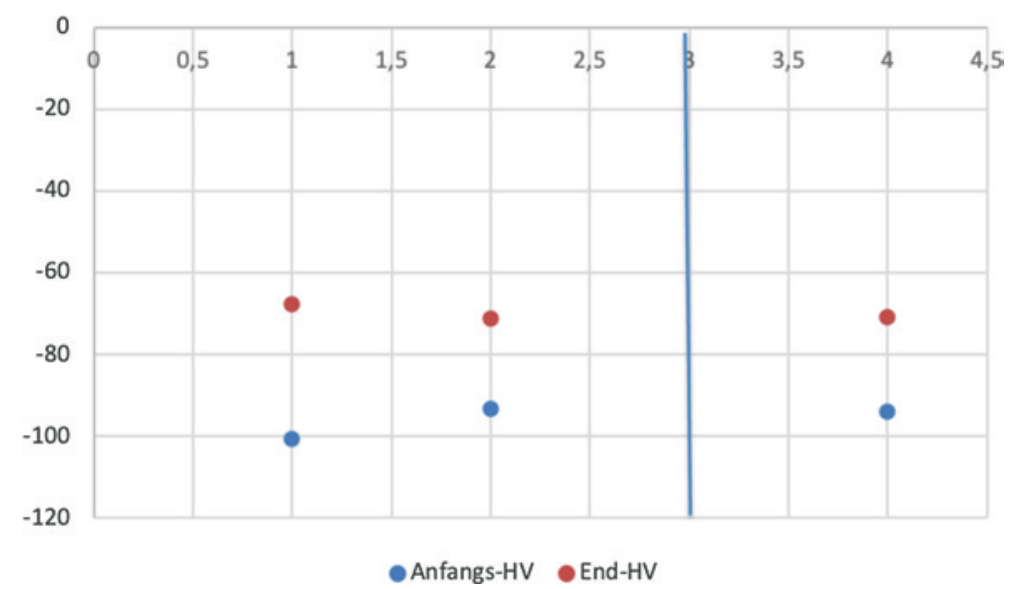

Abb.9.6 Mittlere Tonhörentwicklung der TYMP-FL-Studiengruppe $(\mathrm{n}=79)$. Abszisse: Studiengruppe; Ordinate: Hörverlust in dB; unterer Bildpunkt: Anfangshörverlust; oberer Bildpunkt: Resthörverlust bei der Nachuntersuchung.

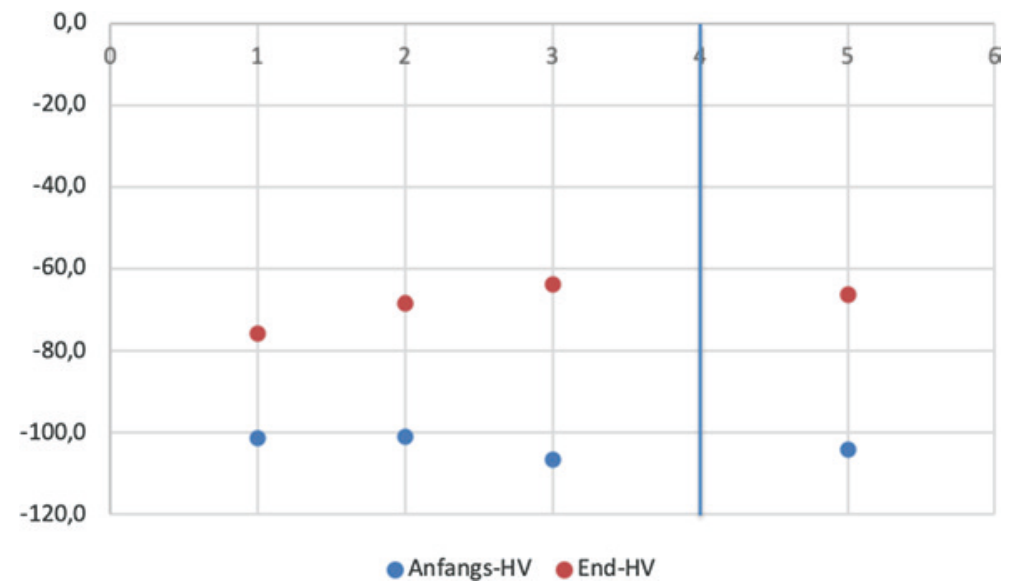

Abb. 9.7 Mittlere Tonhörentwicklung der einzelnen TYMP-SL-Studiengruppen $(\mathrm{n}=247)$. Abszisse: Einzelne Studiengruppen; Ordinate: Hörverluste in $\mathrm{dB}$; rechts neben dem vertikalen Querbalken: gewichtete Mittelwerte aus den einzelnen Studiengruppen in dB; unterer Bildpunkt: Anfangshörverlust; oberer Bildpunkt: Resthörverlust bei der Nachuntersuchung.

nur zurückhaltend und oft erst nach Versagen anderer Behandlungsarten eingesetzt worden war. Insofern können die therapiebedingten Hörgewinne, die jeweils von einem unterschiedlichen Resthörvermögen einsetzen, nur sehr eingeschränkt miteinander verglichen werden. Bezogen auf die Tympanoskopie konnten die Arbeiten von Reineke et al. und Ulk-Mulk et al. zeigen, dass bei präoperativ geringeren Ausgangshörverlusten, die nicht einer Hörminderung vom „severe“, 


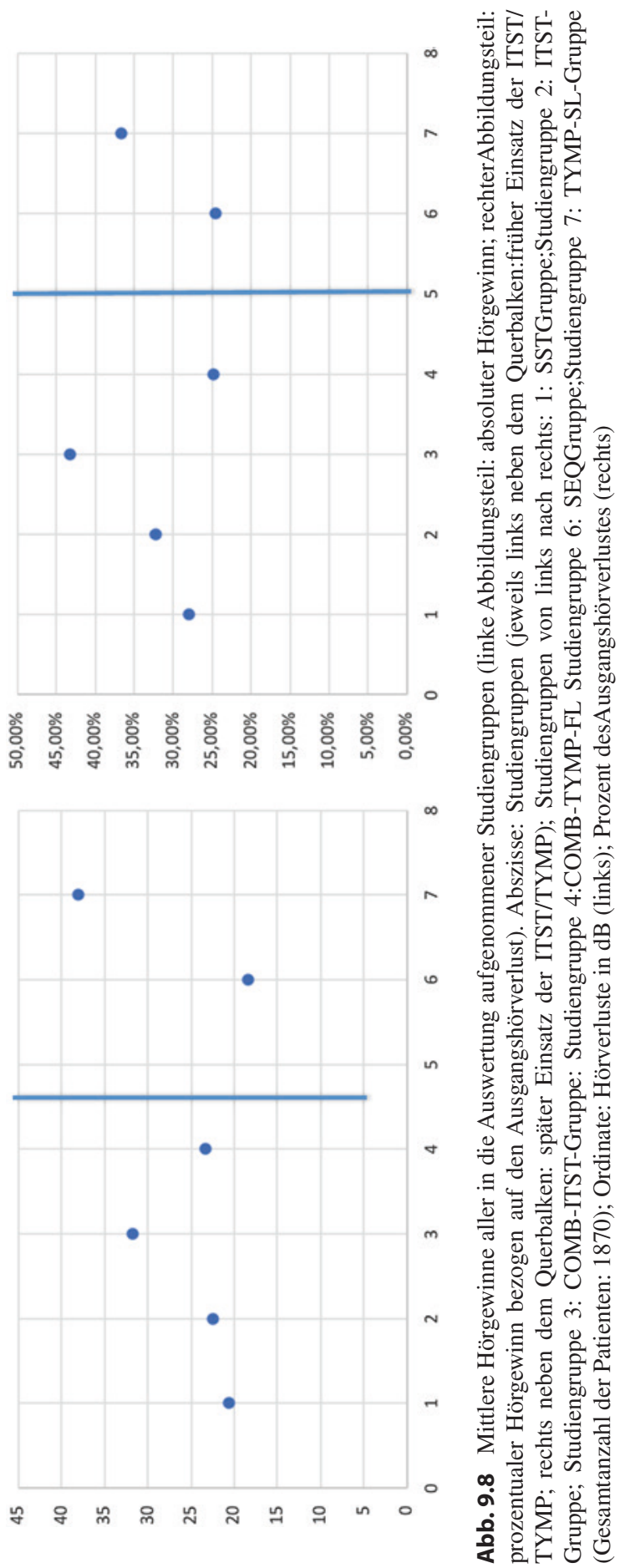




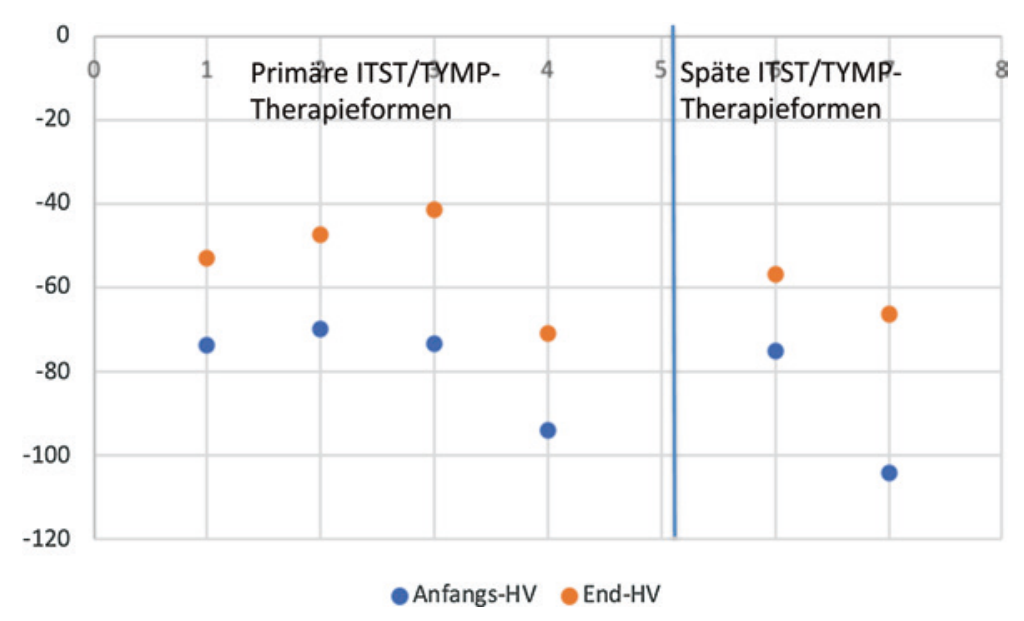

Abb.9.9 Gewichtet gemittelte Hörverluste (Anfangs- und Endwerte) der Patienten bei den einzelnen untersuchten Therapiearten. Abszisse: EinzelneTherapiearten: linkes vertikales Hörverlustpaar: SST-Gruppe; zweites vertikales Hörverlustpaar von links: ITST-Gruppe; drittes vertikalesHörverlustpaar von links: COMB-ITST-Gruppe; vertikales Hörverlustpaar links neben dem Querbalken: COMB-TYMP-FL-Gruppe; erstesvertikales Hörverlustpaar neben dem Querbalken: SEQ-Gruppe; rechtes vertikales Hörverlustpaar: TYMP-SL-Gruppe. Ordinate: Hörverluste in $\mathrm{dB}$; unterer Bildpunkt: Anfangshörverlust; oberer Bildpunkt: Resthörverlust bei der Nachuntersuchung.

„profound“ oder „,total deaf“ Typ entsprachen, auch geringere Hörgewinne erzielt werden konnten, als es bei höheren Ausgangshörwerten der Fall war [78, 101].

\subsubsection{Hörgewinne in den einzelnen Studiengruppen}

Die Patienten mit Tympanoskopie im First-Line-Modus erreichten bei der Nachuntersuchung nicht einen gleich guten Hörlevel, wie er sich bei den verschiedenen mit Steroiden Behandelten eingestellt hatte. Die Hörgewinne waren in den ITST- und TYMP-behandelten Patientenkollektiven, absolut gesehen, etwa gleich stark ausgeprägt, selbst wenn sich die Ausgangshörwerte im Durchschnitt um $>20 \mathrm{~dB}$ unterschieden. Im Second-Line-Modus konnten die Tonhörschwellen der TYMP-SL-Gruppen von einem ungünstigeren Anfangshörwert ausgehend, durch beachtliche Hörverbesserungen bei der langfristigen Followup-Untersuchung die anfänglich bestehende, gravierende Differenz der mittleren Hörverluste zu den Erkrankten, die im SEQ-Verfahren behandelt worden waren, deutlich besser ausgleichen, als es bei der Therapie im First-Line-Modus möglich war. Aus diesen Ergebnissen ziehen wir den Schluss, dass bei einem sehr akzentuierten Ausgangshörverlust auch die frühe Indikation zur Tympanoskopie die Patienten mit der Durchführung der Operation im Vergleich zu den anderen Behandlungsverfahren (systemische oder intratympanale Steroidgabe) keiner erhöhten Gefahr aussetzt. Sollte hingegen eine vorausgehende systemische Gabe von Kortikosteroiden zu keinem befriedigenden Hörerfolg geführt haben, stellt die 


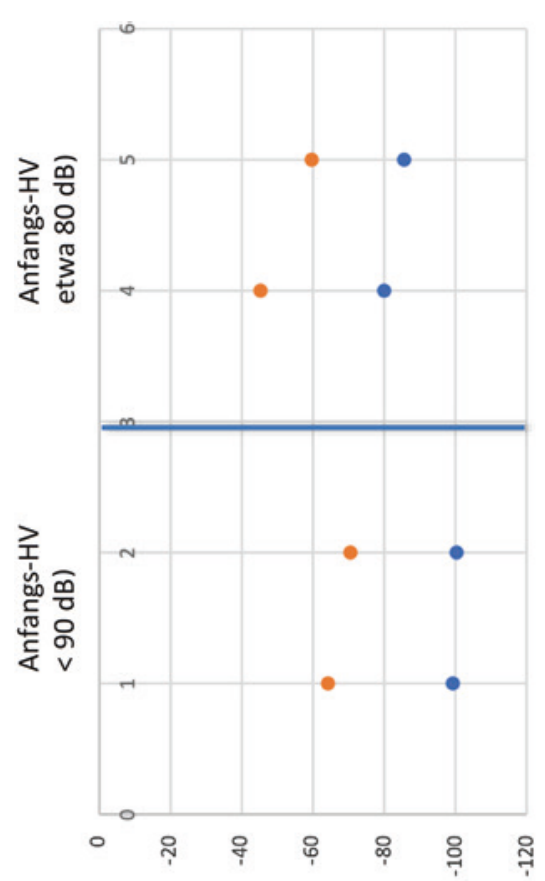

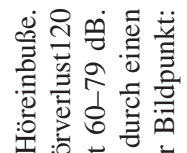

I: :

可

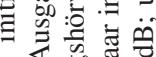

๑ै

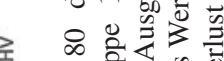

穵

-

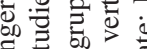

焉

$\because \because \frac{\overline{0}}{\square}$

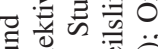

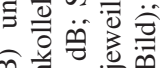

ๆิ

๙

$\wedge \approx$ 可

可

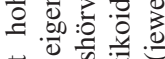

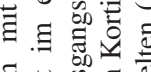

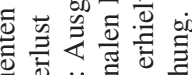

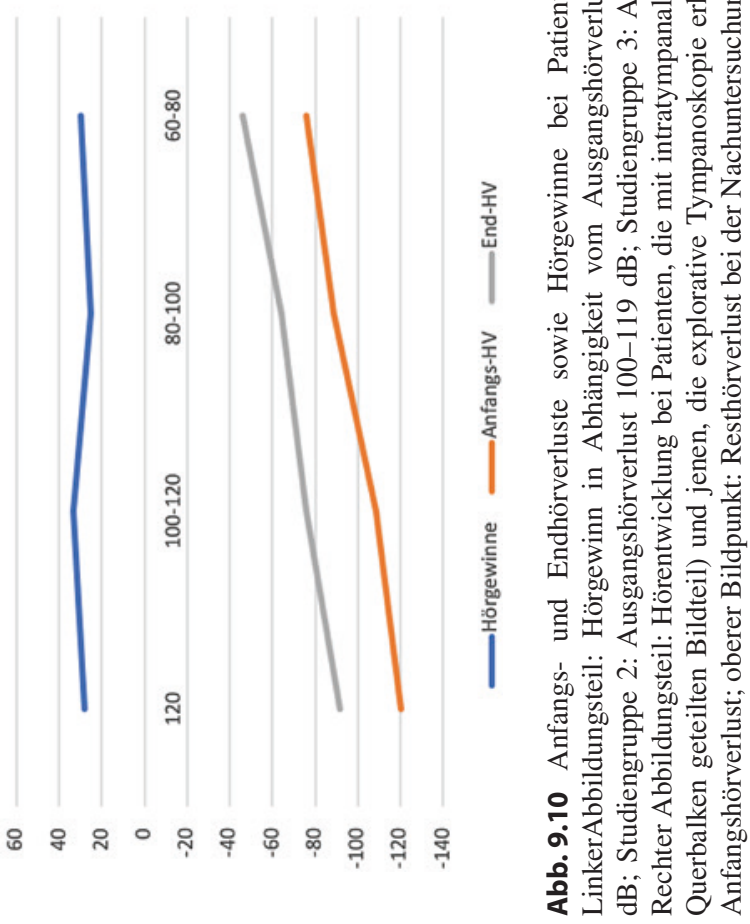


Tympanoskopie als Salvage Therapy ein mehr als respektables Alternativverfahren zur intratympanalen Applikation von Steroiden dar.

\subsubsection{Beziehung der Ausgangshörverluste zu den Hörgewinnen bei einer ITST- bzw. TYMP-Behandlung}

Bisher haben wir in der Literatur nur zwei Studien gefunden, in denen intratympanale Kortikoidinjektionen angewendet und der Frage der Abhängigkeit des Hörgewinns vom Ausmaß des initialen Hörverlustes nachgegangen wurde $[5,90]$. In beiden Studien waren jeweils zwei Subpopulation aus dem Gesamtpatientengut gebildet worden, die eine mit Ausgangshörverlusten von $>90 \mathrm{~dB}$, die zweite beinhaltete Patienten, deren Anfangshöreinschränkungen $<90$ dB lag. Dem haben wir zum Vergleich jene Patienten des eigenen Krankengutes gegenübergestellt, die nach den gleichen Kriterien ausgewertet worden waren (Abb. 8.10).

Bei unseren Erkrankten zeigte sich, dass Patienten mit einem durchschnittlichen Ausgangshörverlust von 100,4 dB (also $>90 \mathrm{~dB}$ ) einen nahezu gleich großen Hörgewinn aufwiesen wie jene, deren initial mittlerer Hörwert deutlich besser war, nämlich 85,6 dB (also $<90 \mathrm{~dB}$ ). Der Hörgewinn der Patienten mit Hörverlust $>90 \mathrm{~dB}$ lag bei 24,6 dB (SAW: $25,7 \mathrm{~dB} ; \mathrm{n}=13$ ), der Hörgewinn von Patienten mit einem Hörverlust $<90 \mathrm{~dB}$ bei $26,4 \mathrm{~dB}$ (SAW: $31,4 \mathrm{~dB} ; \mathrm{n}=21$ ). Vergleichend dazu erreichten bei gemittelten Ausgangshörverlusten von 99,4 dB (also $>90 \mathrm{~dB}$ ) die zusammengefassten Studiengruppen von Battaglia et al. und Suzuki et al. in ihren ITST-behandelten Patientengruppen Hörverbesserungen von 35,3 dB, jene Patienten mit initialen Höreinschränkungen von $80 \mathrm{~dB}$ (also $<90 \mathrm{~dB}$ ) Hörgewinne von $34,8 \mathrm{~dB}[5,91]$. Hier waren ebenso keine nennenswerten Unterschiede in beiden Untersuchungsgruppen nachweisbar. Die Ergebnisse deuten darauf hin, dass bei geringeren Ausgangsverlusten (wie alle mit intratympanalen Kortikoidinjektionen behandelten Patienten gegenüber den mit Tympanoskopie Behandelten in dieser Arbeit zeigten) nicht davon ausgegangen werden kann, dass diese Patienten geringere Hörgewinne erreichen als Patienten mit ausgeprägteren initialen Höreinschränkungen, was gleichermaßen auch im Umkehrschluss gilt. Dies gilt zumindest für Anfangshörverluste, die oberhalb von $80 \mathrm{~dB}$ liegen. Für anfängliche Höreinschränkungen $<70 \mathrm{~dB}$ muss diese Aussage keinen Bestand haben.

Wenn man berücksichtigt, dass bei den spät Tympanoskopierten, bei denen bis zur Operation bereits eine erhebliche Zeit nach dem Hörsturzereignis verstrichen war, die - absolut gesehen - besten Endhörresultate aus allen Therapiegruppen erreicht werden konnten, scheint sich die chirurgische Behandlung noch sehr zu lohnen und sollte vielleicht sogar einer ITST-Therapie vorgezogen werden. Auch wenn bei der Mehrzahl der auf diese Weise Behandelten die Abdeckung des runden/ovalen Fensters keine kausale Therapiemethode darstellte, wie es für diejenigen mit nachgewiesenen Perilymphfisteln der Fall war, erscheint uns dieses Resultat doch beachtlich. 


\subsection{Statistische Beziehungen zwischen einzelnen Studiengruppen mit intratympanalen Kortikoidinjektionen}

In einigen Publikationen, die sich mit intratympanalen Kortikoidinjektionen beschäftigt haben, waren innerhalb einer Studie auch bis zu zwei Vergleichstherapiegruppen mit voneinander unterschiedlichen Behandlungsverfahren mitgeführt worden. Die Autoren haben mit statistischen Verfahren untersucht, ob sich signifikante $(\mathrm{p}<0,05)$ Unterschiede in den Therapieeffekten zwischen den einzelnen Subpopulationen ihres Gesamtkrankengutes ergeben hatten. Das Ergebnis dieser Analysen spiegelt die Tab. 9.10 wieder. Es ergibt sich ein recht uneinheitliches Bild.Wurden von mehreren Autoren parallel zueinander Therapievergleiche vorgenommen (z. B. ITST-Applikation vs. COMB-ITST-Applikation von Kortikosteroiden), konnten in einigen Arbeiten, bei denen Behandlungsmodi miteinander in Beziehung gesetzt wurden, signifikante Unterschiede zwischen den einzelnen Therapieerfolgen registrieren werden, während dies in anderen Studien bei einer gleichartigen Gegenüberstellung der beiden miteinander zu vergleichenden Behandlungsverfahren nicht gelang (Kontroll-/Nulltherapiegruppe vs. ITSTGruppe,SST- vs. ITST-Gruppe, SST- vs. COMB-ITST-Gruppe). Für die Gegenüberstellung der Resultate der SST- vs. der SEQ-Gruppe wie auch der ITSTvs. der SEQ-Gruppe liegt bislang jeweils nur eine Auswertung - die dann jeweils einen signifikanten Unterschied erbrachte -vor, sodass man sich hier noch kein repräsentatives Bild über das Ausmaß der Übereinstimmung resp. Unterschiede zwischen den beiden Patientenklientel verschaffen kann.

Für die mit einer explorativen Tympanoskopie behandelten Patienten liegen, wie bereits beschrieben, noch keinerlei Mitteilungen vor, bei denen innerhalb einer Studie der Vergleich zu anderen Behandlungsverfahren hergestellt wurde. Hier besteht ein deutlicher Nachholbedarf. Nur die Daten aus Vergleichsgruppen innerhalb einer Versuchsreihe einer Untersuchung können einer Metaanalyse zugeführt werden. So haben wir es in der vorliegenden Studie dabei belassen müssen, nur ein systematisches Review erstellen zu können. Im eigenen Patientengut, wo wir die entsprechenden Vergleiche durchführen konnten, hatte die Gegenüberstellung der COMB-TYMP-FL- und der TYMP-SL-Gruppe mit einem p-Wert von 0,55 keinen signifikanten Unterschied bei den Hörgewinnen erbracht.

\subsection{Hörgewinne nach den modifizierten Kanzaki- Kriterien}

\subsubsection{Rekrutierung des Patientengutes}

Bei der Bewertung der Hörerfolge nach den modifizierten Kanzaki-Kriterien standen uns für die ITST-, COMB-ITST- und SEQ-Gruppe jeweils eine, bei den tympanoskopierten Gruppen 3 Patientenkollektive zur Auswertung zur Ver- 
Tab.9.10 Vergleich des Ausmaßes von Therapieeffekten, die bei der Gegenüberstellung zwischen einzelnen Behandlungsverfahren einschließlich einer Nultherapie (Kontrollgruppen) aus Literaturangaben

\begin{tabular}{|c|c|}
\hline Studie & Angabe der Signifikanz \\
\hline \multicolumn{2}{|c|}{ Nulltherapie vs. ITST } \\
\hline Moon & s \\
\hline Yang (2013) & ns \\
\hline Yang (2015) & s \\
\hline \multicolumn{2}{|l|}{ SST vs. ITST } \\
\hline Dispenza & ns \\
\hline Jiang & ns \\
\hline Kosaykov & $\mathrm{s}$ \\
\hline Rauch & ns \\
\hline Lee (2015) & ns \\
\hline \multicolumn{2}{|c|}{ SST vs. COMB-ITST } \\
\hline Battaglia & s \\
\hline Gundogan & $\mathrm{s}$ \\
\hline Jiang & ns \\
\hline Lee(2015) & ns \\
\hline \multicolumn{2}{|l|}{ SST vs. SEQ } \\
\hline Moon & s \\
\hline \multicolumn{2}{|l|}{ ITST vs. SEQ } \\
\hline Yang & s \\
\hline
\end{tabular}

fügung (Tab. 9.3). Bei den Behandelten mit intratympanalen Kortikoidinjektionen mussten 4 Studiengruppen aus der Auswertung ausgeschlossen werden, da entweder keine Zeitangabe zur Nachuntersuchung bestand oder diese direkt postoperativ erfolgt war $[13,18,92,111]$. Wir mussten aufgrund der wenigen verbleibenden Studiengruppen $(n=3)$ auf eine Unterteilung der im FL- und SLModus Therapierten verzichten und diese beiden Therapieformen in der weiteren Auswertung zusammenfassen.

\subsubsection{ITST-Gruppe}

Die auf verschiedene Weise mit intratympanalen Kortikoiden Therapierten erreichten im gewichteten Mittel in 25,3\% der Fälle komplette und in 38,5 \% markante Hörgewinne $(n=253)$ (Tab. 9.3 und Abb. 9.11). Fasst man die drei Hörverbesserungsgruppen zusammen, konnte bei 63,6 \% der Patienten Hörgewinne von $>30 \mathrm{~dB}$ (Typen 1 und 2) dokumentiert werden. 


\subsubsection{TYMP-Gruppe}

Im eigenen Patientengut konnten 44,8 \% der Betroffenen $(\mathrm{n}=19)$ Hörgewinne von $>30 \mathrm{~dB}$ erreichen, wobei 10 Patienten $(24,3 \%)$ als vollständig erholt

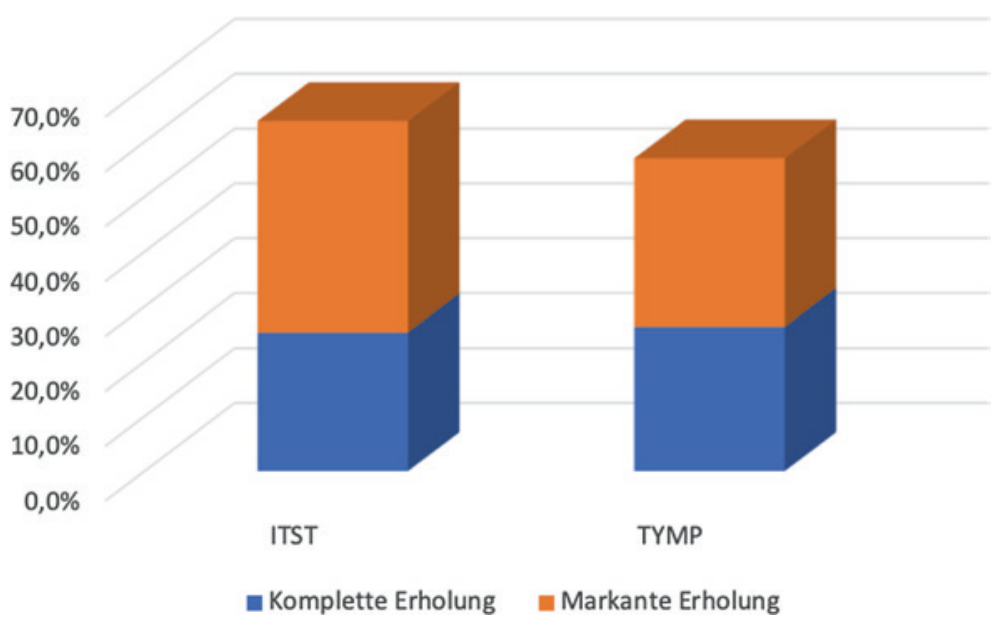

Abb.9.11 Hörgewinne in einzelnen Studiengruppen nach Kanzaki-Kriterien. Abszisse: Einzelne Studiengruppen: linke Säule: Patienten mit intratympanalen Kortikoidsteroidinjektionen (ITST-, COM-ITST- und SEQ-Gruppen), rechte Säule: Patienten mit explorativer Tympanoskopie (COMB-TYMP-FL- und.TYMP-SL-Gruppen); Ordinate: Komplette (blauer Säulenanteil) und markante Hörgewinne (roter Säulenanteil) in Prozent

(Typ1) angesehen werden können (Tab. 9.8). Bei 9 Erkrankten (22\%) war es noch zu Hörgewinnen von $>30 \mathrm{~dB}$ (Typ 2) gekommen, ohne dass eine komplette Hörerholung erreicht worden war. $29,3 \%$ der Patienten $(n=12)$ erzielten geringe Hörgewinne zwischen $10 \mathrm{~dB}$ und $30 \mathrm{~dB}$. Bei 5 Operierten (12,2\%) war keine Hörveränderung nach der Tympanoskopie eingetreten. Zudem waren bei weiteren 5 Behandelten (12,2\%) langfristig Hörverschlechterung gegenüber dem präoperativen Wert aufgetreten.

Stellt man im eigenen Krankengut die früh Tympanoskopierten den spät Operierten gegenüber, so erreichten in der FL-Gruppe $40 \%$ der Patienten eine komplette bzw. markante Hörverbesserungen (komplette Hörerholung $40 \%$; markante Hörerholung: $0 \% ; n=10$ ), während dies sogar für $70 \%$ der Patienten in der SL-Gruppe (komplette Hörerholung: $60 \%$; markante Hörerholung: $10 \%$; $\mathrm{n}=10$ ) zutraf.

Im gemittelten Vergleich der drei chirurgisch therapierten Studiengruppe erreichten 57,0 \% der Erkrankten einen vollständigen oder markanten Hörgewinn (Abb. 9.11). Bei 26,3 \% hatte sich eine komplette Hörerholung eingestellt, 30,7 \% erreichten nur geringe Hörgewinne. 


\subsubsection{Vergleich der Kanzaki- mit den Siegel-Kriterien}

Die Kanzaki-Kriterien scheinen uns für die Auswertung der vorliegenden Studiengruppen deutliche Vorteile gegenüber den sog. Siegel-Kriterien zu besitzen [35, 82]. Bei dem Verfahren nach Siegel fließen die nach der Therapie erreichten Endhörverluste in die Bewertung mit ein. Da bei den Tympanoskopierten deutlich höhere Anfangshörverluste bestanden als bei den Kortikoidtherapierten, müssten nach Siegel Erstere erheblich höhere Hörgewinne erreichen, um in dieselbe Erfolgskategorie eingeordnet zu werden, wie diejenigen, die mit Glukokortikoiden behandelt wurden. Dies würde zu einer erheblich eingeschränkten Chancengleichheit in der Bewertung der im Vergleich zueinander stehenden Studiengruppen führen. Aus diesem Grund haben wir auf die Analyse des Datenmaterials nach der Siegel-Methode verzichtet.

\subsubsection{Vergleich der Ergebnisse in den Studiengruppen}

Legt man der Bewertung der Hörerfolge die Kanzaki-Kriterien zugrunde, erreichten die Patienten mit den intratympanalen Kortikoidinjektionen mit 63,8 \% die höchste Rate an Werten, die dem Typ 1 und Typ 2 der Kanzaki-Kriterien, also der vollständigen Erholung resp. dem Erreichen markanter Hörverbesserungen zugeordnet werden konnten. Patienten, die eine Tympanoskopie erhielten, lagen mit einem Prozentwert von $57 \%$ geringfügig unter dieser Quote.

\subsection{Weitere angestrebte Studien}

Eine denkbare Alternative in der Behandlung des Hörsturzes würde der sukzessive Einsatz zuerst der ITST und bei unzureichendem Therapieerfolg nachfolgend der Tympanoskopie bedeuten. Dieser Behandlungsansatz ist unseres Wissens bislang noch nicht verfolgt worden.

\subsection{Ausblick}

Auch wenn in dem vorliegenden Beitrag, insbesondere im Hinblick auf die Tympanoskopiestudien und den hier angegangenen Fragestellungen, einige der streng wissenschaftlichen Analysekriterien nicht erfüllt waren, erbrachte der aktuelle Vergleich der intratympanalen Steroidapplikation mit der explorativen Tympanoskopie unter Einschluss der Abdeckung der runden/ovalen Fensternische mit körpereigenem Bindegewebe Ergebnisse, die nahelegen, dass mit dem Einsatz des letztgenannten Therapieverfahrens kein gravierender Fehler gemacht wird. Für eine valide Unterscheidung des Effektes beider Vorgehensweisen wäre aus unserer Sicht eine prospektive, idealerweise auch randomisierte und verblindete 
Studie notwendig, die darüber hinaus möglichst an einem einzigen Studienort vorgenommen werden sollte. Bis aus solchen Untersuchungen statistisch verwertbare Daten vorliegen, können die hier vorgelegten Resultate allenfalls als Ansporn dienen, den hier beobachteten Phänomenen weiter auf den Grund zu gehen.

\subsection{Zusammenfassung}

Die Applikation von Steroiden in vielfältiger Form und die explorative Tympanoskopie mit Obliteration des runden/ovalen Fensters sind die geläufigsten Verfahren, mit denen die akut aufgetretene, idiopathische sensorineurale Hörminderung, insbesondere wenn sie hochgradig auftritt, weltweit behandelt wird. In der vorliegenden Studie werden, zusammengetragen aus Literaturberichten und der Hinzufügung eines eigenen Patientengutes, die unterschiedlich gebräuchlichen Therapieformen miteinander verglichen. Insgesamt konnten 23 Publikationen (34 Studiengruppen), die sich einerseits mit der systemischen Gabe von Glukokortikoiden und/oder intrataympanalen Steroidinjektionen beschäftigen und andererseits 4 Arbeiten (5 Studiengruppen) sowie 3 eigene Studiengruppen, bei denen eine Tympanoskopie mit Abdichtung des runden/ovalen Fensters durchgeführt worden war, in die Auswertung einbezogen werden. Insgesamt fanden 2117 Patienten, die zwischen 2006 und 2015 behandelt worden waren, Eingang in dieses systematische Review. Die Hörverluste wurden tonschwellenaudiometrisch bestimmt und ein mittlerer Hörverlust $\mathrm{PTA}_{4}(0,5,1,2$ und $4 \mathrm{kHz}$ oder $0,5,1,2$, $3 \mathrm{kHz})$ oder PTA $_{5}(0,25,0,5,1,2,4 \mathrm{kHz})$ eruiert. Zusätzlich wurden die Hörgewinne ermittelt, die bei Anwendung der Kanzaki-Kriterien erreicht worden waren. Entsprechend der Form, in der die Steroide verabreicht wurden, konnten verschiedene Subgruppen gebildet werden. Einige Patienten erhielten nur intratympanale Kortikoidinjektionen (ITST-Gruppe; $\mathrm{n}=525$ ). Intratympanale Kortikoidinjektionen wurden in einer zweiten Untergruppe als Primärtherapie synchron zusammen mit systemischen Kortikoidgaben verabreicht (COMB-ITSTGruppe; $n=437$ ). Die intratympanalen Kortikoidgaben wurden in einer dritten Subgruppe sequenziell nach der systemischen Gabe von Steroiden appliziert (SEQ-Gruppe; $n=306$ ). Bei den Tympanoskopien wurde zwischen einem FirstLine-Einsatz (COMB-TYMP-FL-Gruppe; $n=79$ ) und Second-Line-Einsatz (TYMP-SL-Gruppe; $\mathrm{n}=247$ ) der Operation bezogen auf die systemische Kortikoidgabe unterschieden.

Innerhalb der einzelnen Untersuchungsgruppen zeigten sich jeweils erhebliche Schwankungen sowohl bezüglich der Anfangs- wie auch der Endhörverluste, die im Rahmen einer Nachuntersuchung dokumentiert wurden. Alle mit intratympanalen Kortikoidinjektionen behandelten Patienten wiesen jeweils geringe Ausgangshörverluste auf als die mittels Tympanoskopie Therapierten. Den größten absoluten Hörgewinn erreichten die Patienten, bei denen eine verzögerte (Second-Line-)Tympanoskopie durchgeführt worden war (TYMP-SL: 38,1 dB). Die verschiedenen Gruppen der mit intratympanalen Kortikodinjektionen 
Behandelten erreichten Hörverbesserungen zwischen 18,4 dB (SEQ-Gruppe) und $31,7 \mathrm{~dB}$ (COMB-ITST-Gruppe). Die operierten Patienten erzielten im Second-Line-Modus, obwohl sie von einem schlechteren Hörlevel ausgingen, Endhörverluste, die nahe an diejenigen heranreichten, die mit intratympanalen Kortikoidinjektionen erzielt worden waren. Der prozentuale Hörgewinn, gemessen am Ausgangshörverlust, lag in der COMB-ITST-Gruppe mit 43,3 \% am höchsten, gefolgt von der TYMP-SL-Gruppe mit 36,6\% und der ITST-Gruppe mit 32,2\%.

Erste Ergebnisse deuten darauf hin, dass die Höhe des Anfangshörverlustes weder bei den ITST-Behandelten noch bei den Patienten mit Tympanoskopie einen deutlichen Einfluss auf die Höhe des langfristig erreichten Hörgewinns nimmt, solange der Ausgangshörverlust $>70 \mathrm{~dB}$ betrug.

Bei Publikationen, die die intratympanalen Kortikoidinjektionen zum Thema hatten, zeigte sich in den Fällen, in denen mehrerer Studiengruppen mit unterschiedlichem Behandlungsregime parallel laufen gelassen und auf ihre Therapieerfolge untersucht wurden, ein uneinheitliches Bild bezüglich der Beantwortung der Frage, ob einer der angewendeten Behandlungsmodi den anderen statistisch signifikant überlegen ist.

Die Tympanoskopie mit Obliteration der runden/ovalen Fensternische mit autologem Bindegewebe stellt ein Therapieverfahren dar, das respektable Ergebnisse bei hochgradigen Hörverlusten und selbst Taubheiten aufzuweisen hat, insbesondere wenn die Operation verzögert im Second-Line-Modus durchgeführt wird. Für ihre Anwendung als Primärtherapie (First-Line-Modus) zusammen mit systemischen Kortikoidgaben liegen bislang noch zu wenige Erfahrungen vor, um diese Einsatzform der Tympanoskopie bereits jetzt uneingeschränkt empfehlen zu können. Zukünftige prospektive Studien, möglichst unter Mitführung von Kontrollgruppen, sollten zur Klärung dieser Fragestellung hilfreich sein.

Open Access Dieses Kapitel wird unter der Creative Commons Namensnennung 4.0 International Lizenz (http://creativecommons.org/licenses/by/4.0/deed.de) veröffentlicht, welche die Nutzung, Vervielfältigung, Bearbeitung, Verbreitung und Wiedergabe in jeglichem Medium und Format erlaubt, sofern Sie den/die ursprünglichen Autor(en) und die Quelle ordnungsgemäß nennen, einen Link zur Creative Commons Lizenz beifügen und angeben, ob Änderungen vorgenommen wurden.

Die in diesem Kapitel enthaltenen Bilder und sonstiges Drittmaterial unterliegen ebenfalls der genannten Creative Commons Lizenz, sofern sich aus der Abbildungslegende nichts anderes ergibt. Sofern das betreffende Material nicht unter der genannten Creative Commons Lizenz steht und die betreffende Handlung nicht nach gesetzlichen Vorschriften erlaubt ist, ist für die oben aufgeführten Weiterverwendungen des Materials die Einwilligung des jeweiligen Rechteinhabers einzuholen.

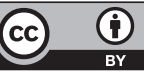

